\section{Effects of item discriminability in mixed-list presentation of paired associates}

\author{
GUY J. JOHNSON \\ University of British Columbia, Vancouver 8, B.C., Canada
}

The present study investigated the effects of intersubset discriminability on the performance of strongly and weakly associated pairs presented under a mixed-ist procedure. In the low-discriminability condition, the two subsets were presented in the same color, while in the high-discriminability condition, each subset appeared in a different color. Thirty-six Ss were assigned to each condition. Performance measures in terms of number of correct responses and number of trials to perfect recitation indicated facilitation effects for both strong- and weak-association pairs under the high-discriminability condition. The results were discussed in terms of intralist generalization and differentiation.

A number of recent studies have focused upon characteristics of performance on paired-associate items under the condition of mixed-list (ML) presentation. Among the findings is the result that performance on a given set of items varies directly as a function of the initial associative strength of other pairs presented in the same list (Johnson \& Penney, 1966; Underwood \& Schulz, 1960). Performance on a given set of strong-association pairs tends to be better where the items are presented in an unmixed list (UL) of strong associations than in an ML where the items are interspersed with low-association pairs. On the other hand, items initially low in associative strength are learned more easily where they are mixed with strong associations than where they are presented along with other weak associations in a UL.

The interaction between initial strength of association and type of design has been shown to be appropriate tc performance at a transfer task, as well as where Ss learn a single list (Johnson, 1970). Performance on $\mathrm{AB}-\mathrm{ABr}$ (negative paradigm) items is better in a $\mathrm{ML}$ that contains a subset of $\mathrm{AB}-\mathrm{AB}^{\prime}$ (positive paradigm) items than in an $\mathrm{AB}-\mathrm{ABr}$ UL. Further, ML performance on the subset of $A B-A B^{\prime}$ pairs is impaired, at least early in training, relative to performance on those items presented in an unmixed $A B-A B^{\prime}$ list.

One interpretation of the effects of $M L$ vs UL design with high- and low-association pairs has been presented elsewhere (Johnson, 1970). The analysis is based upon a hypothesized inverse relationship between initial associative strength of to-be-learned pairs and the magnitude of intralist generalization tendencies. That is, the amount of intralist interference generated by a subset of weakly associated items is considered to be greater than that produced by a subset consisting of an equal number of highly associated pairs. Items initially high in associative strength may be less likely to produce response generalization tendencies, since at the beginning of training, response terms are already more strongly associated with appropriate than with inappropriate stimuli. For a subset of low-association pairs, however, no such pretraining response differentiation obtains, and response terms are just as likely to be elicited by inappropriate as by appropriate stimuli.

Performance differences on a given set of test items in ML vs UL may be viewed as reflecting differential strength of intrusion tendencies generated by the respective subsets with which test pairs are combined under the two conditions. Performance on strongly associated items is expected to be better in UL, since the amount of interference generated by other strong associations is less than that resulting from the subset of low associations in ML. Similarly, ML performance on low-association pairs is expected to be superior to UL, due to the lesser amount of interference produced by interspersed high associations in ML relative to that provided by other low associations in UL.

If the mixed-association list effect is in fact related to the strength of intersubset generalization tendencies, an important factor would seem to be the degree of discriminability of the subsets of items presented in ML. The present study was designed to explore the effects of such a manipulation. Strongly and weakly associated subsets of items were presented in ML under two conditions of discriminability. Under one condition the two subsets appeared in the same color (SC), while in another condition each subset was presented in a different color (DC). Performance on the DC list was expected to be superior, due to the effects of interitem perceptual discriminability in reducing the strength of potential intrusion tendencies across subsets.

\section{MATERIALS}

Each S learned a 16-item paired-associate list constructed of two-syllable adjectives adopted from Melton (1940). The subset of 8 items of initial high-associative strength consisted of the following pairs: perfect-faultless, a brupt-sudden, wicked-evil, ready-prepared, timid-bashful, complete-thorough, awkward-clumsy, and absent-missing. The subset of weakly associated items contained the following eight pairs: yawning-little, feline-distant, joyous-adept, vocal-nomad, twisted-hidden, wholesome-grouchy, uphill-barren, and stubborn-careful. Interitem similarity was as low as could be achieved by careful inspection of the items.

The two subsets of items were interspersed randomly and presented under four list conditions. List DC1 presented strong associations in red and weak associations in black, while List DC2 represented the counterbalanced arrangement of the subset-color combination. In SC1 all items appeared in red, and in SC2 all items appeared in black.

\section{SUBJECTS}

Ss were 72 female undergraduate students from psychology courses who served to fulfill a class requirement. No $S$ had previously participated in a verbal learning experiment. Assignment to experimental conditions was conducted in blocks of four Ss each. A predetermined random order of the four conditions was employed for each block.

\section{PROCEDURE}

Immediately after receiving standard instructions for paired-associate learning, Ss were given a maximum of 20 trials at the task. Training was terminated for Ss reaching a criterion of two successive errorless trials or at the end of 20 trials for Ss who failed to achieve this criterion. Perfect scores were assigned to Ss on postcriterial trials.

The lists were typed in uppercase letters on white glazed-cloth tape and presented on a standard Stowe memory drum at a $2: 2$ rate with a 4-sec intertrial interval. Three different random orders of the 16 pairs were used to control for serial effects.

Number of correct anticipations over 20 trials and number of trials to first perfect recitation of the list were recorded for each of the four conditions. Data from the two conditions within each level of the SC-DC variable were combined, since preliminary analysis indicated no differences in 


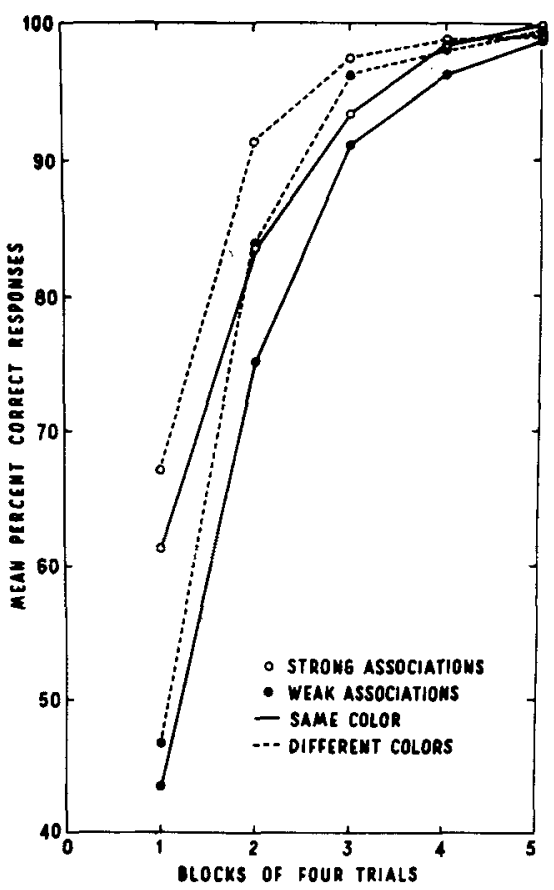

Fig. 1. Mean percent correct responses on strong and weak associations as a function of intralist discriminability.

performance attributable to the effects of color, $F(1,70)<1$.

\section{RESULTS}

Performance curves for SC and DC groups on strongly and weakly associated pairs are shown in Fig. 1. Data are expressed in terms of mean percentage correct anticipations over 20 trials in blocks of four trials each. On both highand low-association items, performance was consistently better under the DC than under the SC condition. Mean number of correct responses on strong associations was 145.56 for $\mathrm{DC}$ and 140.03 for $\mathrm{SC}$. On weakly associated pairs DC and SC conditions yielded respective means of 136.05 and 129.86 correct responses. Mean total correct anticipations was 281.61 for DC and 269.89 for SC. Analysis of variance indicated significant effects for the list variable $[F(1,70)=5.12, p<.05]$ and for strong vs weak associations $[F(1,70)=49.73, p<.001]$. The effect of $S C$ vs $D C$ did not differ as a function of item associative strength, $\mathrm{F}(1,70)<1$.

Mean number of trials to first perfect recitation of the strongly associated subset was 5.86 for $\mathrm{DC}$ and 8.28 for $\mathrm{SC}$ $[F(1,70)=11.23, p<.01]$. The weakly associated subset was learned to this criterion in 7.64 and 9.33 trials, respectively, by $\mathrm{DC}$ and $\mathrm{SC}$ groups $[F(1,70)=4.16, p<.05]$. First errorless recitation of the entire list was achieved by the $\mathrm{DC}$ group at 8.61 and by the SC group at 10.33 mean trials $[F(1,70)=3.72$, $.10>p>.051$.

\section{DISC'USSION}

The results suggest that the discriminability among different subsets of items presented in ML may be a factor determining the extent to which performance on a given subset of pairs is influenced, relative to $\mathrm{UL}$, by the presence of other types of items in the list. This discriminability factor may be expected to limit the generality of the contention that, relative to $\mathrm{UL}, \mathrm{ML}$ performance on a particular subset will be facilitated where the pairs are combined with less-difficult items and inhibited where they are presented with more-difficult pairs. Where intersubset differentiation is relatively complete, generalization tendencies might be limited to those generated by items within each ML subset. Such a reduction in the number of potential intrusions compared to UL would be likely to influence performance on $\mathrm{ML}$ subsets in much the same manner as would a reduction in list length.

Some degree of intersubset discriminability is inherent in certain designs involving $M L$ presentation of items representing different types of transfer paradigms. Control (AB-CD) pairs, for example, may be discriminable from other $\mathrm{ML}$ subsets (such as $\mathrm{AB}-\mathrm{AB}^{\prime}, \mathrm{AB}-\mathrm{AB}_{\mathbf{r}}$, or $A B-A C$ ) from the beginning of second-list training, since C-D items appear only in the transfer task. Such interpair distinctiveness may act to decrease the strength of intersubset generalizations between control pairs and other ML subsets. Thus, where items of an initial high-associative strength (e.g., AB-AB' pairs) are presented along with control items, ML vs UL performance on $A B-A^{\prime}$ pairs may reflect the effects of two opposing factors. The greater difficulty of $A B-C D$ pairs relative to those with which $A B-A B^{\prime}$ test pairs are mixed under UL would be expected to inhibit ML performance. On the other hand, the greater discriminability of the subset of test items in $M L$ is likely to operate in favor of $M L$ performance on $A B-A B^{\prime}$ pairs. Performance on control items would be predicted to show a clear superiority under the ML condition, since both factors would tend to favor ML performance. That is, the subset of $A B-A B^{\prime}$ pairs in $M L$, compared to the subset of nontest items in $A B-C D U L$, is less difficult as well as more discriminable from $A B C D$ test items.

Implications of the above considerations concerning the effects of intersubset discriminability on ML-UL transfer appear to provide an explanation of the results of Johnson \& Penney (1965) with regard to performance on $\mathrm{AB}-\mathrm{CD}$ and $\mathrm{AB}-\mathrm{AB}_{\mathrm{r}}$ items under ML and UL conditions. The results provide only partial support for the assumption that the direction of ML-UL separation varies with initial associative strength, since performance on AB-CD pairs was not inhibited, relative to $\mathrm{UL}$, by the inclusion of more difficult pairs in the list. However, this finding may reflect the effects of an intersubset discriminability based upon first-vs second-list distinctions. Such interpair differentiation might have decreased the strength of potential intrusion tendencies generalized to the set of $\mathrm{C}-\mathrm{D}$ pairs in $\mathrm{ML}$ and thereby reduced ML vs UL differences on those items. The markedly superior performance on $\mathrm{AB}-\mathrm{AB}_{\mathrm{r}}$ items in $M L$ is in agreement with the present considerations, since the differential difficulty of pairs with which they were presented in ML vs UL, as well as the intersubset discriminability factor, would be expected to operate in favor of the ML condition.

Battig (1966) has shown that performance on $\mathrm{AB}-\mathrm{AC}$ items, relative to that on control pairs, increases as the proportion of control pairs is increased in an $A B-A C / A B-C D$ ML. This finding perhaps further indicates the effects of the two intralist factors under discussion. As the proportion of C-D pairs in the second list is increased, the strength of generalization tendencies interfering with AC performance is likely to be reduced as a result of the differences in difficulty of $C-D$ and $A-C$ pairs. Further, as the proportion of $A-C$ pairs is decreased, the number of potential intrusions generated within the subset of $A-C$ items is decreased. Both of these factors would be expected to influence performance on A-C items in the direction of the observed results.

\section{REFERENCES}

BATTIG, W. F. A shift from "negative" to "positive" transfer under the A-C paradigm with increased number of C-D control pairs in a mixed list. Psychonomic Scjence, 1966, 4, 421-422.

JOHNSON, G, J. Systematic strategies and transfer effects with mixed- and unmixed-list designs. Psychological Reports, 1970, 26, 475-484.

JOHNSON, G. J., \& PENNEY, R, K. Transfer effects of mixed and unmixed list designs in paired associate learning of children. Psychonomic Science, 1965, 2, 171-172.

JOHNSON, G. J., \& PENNEY, R. K. The effect of mixed and unmixed list designs in paired associate learning. Journal of Verbal Learning \& Verbal Behavior, 1966, 5, 234-239.

MELTON, A. W. Materials for use in experimental studies of learning and retention of verbal habits. Mimeographed manuscript, University of Missouri, 1940.

UNDERWOOD, B. J., \& SCHULZ, R. W. Response dominance and rate of learning paired associates. Journal of General Psychology, 1960, 62, 153-158. 